\title{
Research on Ancestral Hall Stages in Meishan Area: A Case Study of the Lei family's ancestral hall stage in Changtang Town, Anhua County
}

\author{
Yangzi Qiu, a, Feihu Chen ${ }^{2, b}$ \\ ${ }^{1}$ School of Design, Hunan University, Changsha City, Hunan Province, China \\ ${ }^{2}$ School of Architecture, Hunan University, Changsha City, Hunan Province, China \\ a83211552@qq.com, ${ }^{b} 188000786 @ q q . c o m$
}

Keywords: Meishan, ancestral hall stage, architectural form, decorative art

Abstract. Based on historical background of Meishan area and ancestral hall stages in Meishan, this paper investigated the Lei family's ancestral hall stage in Changtang town, Anhua county as a typical case, analyzed its architectural form and characteristics of decorative art, and discussed its value and significance as one of the few ancient stages in ancestral hall in Meishan area of China.

\section{Introduction}

Some scholars believe that Meishan culture has been a primitive form of civilization and culture with simple folkway and mysterious color in the central area of Hunan province since ancient times [1]. It can be traced back to the Stone Age, more than ten thousand years ago. "Meishan area used to be isolated from the Central Plains, and it borders on Tan (Tanzhou, present Changsha, Hunan) in the east, Shao (Shaozhou, present Shaoyang, Hunan) in the south, Chen (Chenzhou, present Yuanling, Hunan) in the west, Ding and Li (Dingzhou, present Changde, Hunan) in the north, and Meishan is surrounded by these areas" according to the records in Meishandong, History of Song Dynasty [2]. Today it refers to the region of Zijiang River Basin from southwest to northeast- Xuefeng Mountain area between Xiang River and Yuan River, which is south of Dongting Lake and north of Nanling Mountain. This is the narrow definition for Meishan area in terms of geographical scope. I believe that Meishan area, in a broad sense, generally refers to the areas which are characterized with Meishan culture or influenced by Meishan culture.

In Meishan area, people lived together based the relationship by blood at the beginning, and clan became the social existence to maintain such a relationship. Ancestral hall is a public building for sacrifices to ancestors by clan. In the feudal society, traditional Chinese opera was usually a typical sacrificial offering. Therefore, when clansmen with the same surname built the ancestral hall, they also built the stage inside it, which could be used for the performance of traditional Chinese opera to commemorate their ancestors and pray for blessing. At present, well-preserved ancient stages in Meishan area are generally in ancestral halls. The Lei family's ancestral hall stage in Changtang Town, Anhua County is selected as a case study in this paper to enhance our understanding of ancestral hall stages in Meishan area (Fig.1).

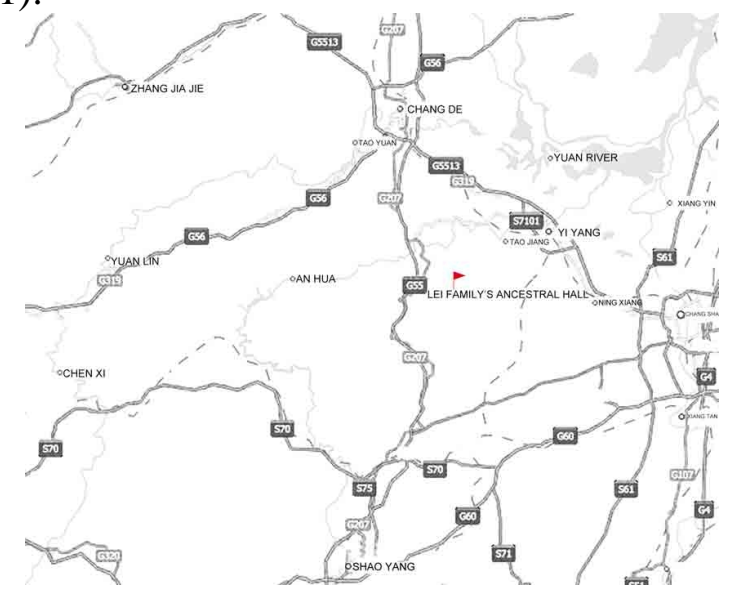

Fig.1: Map of Meishan area and location of Lei family's ancestral hall 


\section{Evolution of Lei family's ancestral hall stage}

Lei family's ancestral hall stage at Changtang Town, Anhua County was initially established in 1874. According to an elder of the clan, due to the migration policy of "immigrating to Hunan from Jiangxi" [3]. In the late Yuan and early Ming dynasties, a large amount of immigrants from Jiangxi flew to Hunan during this period and Lei Family was among them. In 2007, Lei family spontaneously renovated their ancestral hall by organizing a donation. At present, Lei family's ancestral hall stage is the best well-preserved ancient stage in Anhua County and now it is a cultural relic protection place at county level.

\section{Layout of Lei family's ancestral hall stage}

Lei family's ancestral hall stage, a typical ancient stage, is established attached on the ancestral hall. The layout of the ancestral hall is designed with "two entrances with one courtyard", facing south, which is strictly symmetric with the south-north axis as the centerline. There are several parts along the axis from south to north, which are the entrance (including the stage), courtyard, and sacrifice hall (Fig. 2). The architectural plan is about 25.6 meters wide. The stage is connected with the entrance, so that "ancestors" can see the performance conveniently. The stage is in the face of sacrifice hall. Therefore, sacrifice hall is the best place to watch performance. Wing-rooms are arranged at two sides of courtyard, and outside of the wing-rooms is a corridor which is about 0.85 meters wide. Above the wing-rooms is an open auditorium at the second floor, which is about 5.6 meters wide and 16.5 meters long (Fig. 3). The wing-rooms, sacrifice hall, and courtyard constitute a standard quadrangle courtyard and the acreage of courtyard is about 218.4 square meters, which can accommodate a large number of audiences. The plan of stage looks like a rectangle, which is about 8.2 meters long and 5.2 meters wide with pillars in the four corners. Wallboard is set up in front of rear pillars to divide the stage into proscenium (performance area) and backstage (accompaniment area). To make the stage more accessible to actors, a door is opened at each side of the wallboard, the door in the right side is for actors to enter on the stage, which is called "Chujiang", and the door in the left side is for actors to exist, which is called "Ruxiang". There are dressing rooms in both the left and the right sides of the stage. To highlight the importance of the stage, it is not in parallel with dressing rooms but exceeds 3.3 meters forward. The stage and the dressing rooms together take on a "convex shape". Side doors are opened in the enclosing wall at the two sides of the sacrifice hall, so as to make it convenient for audiences to come in and go out.

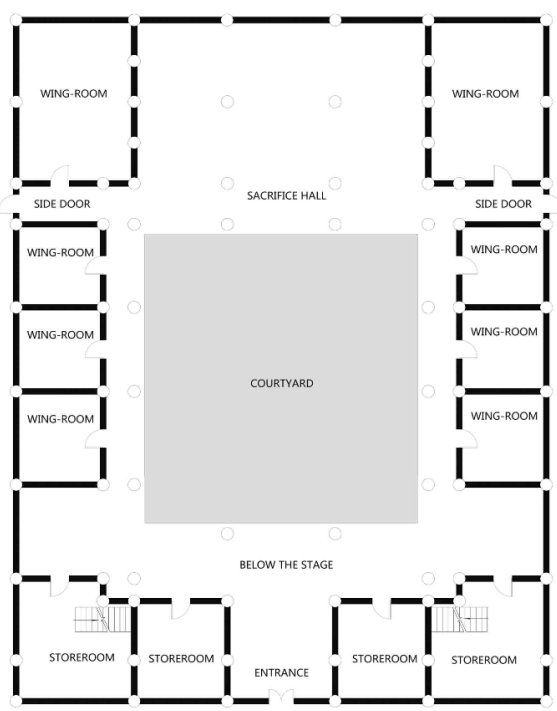

Fig.2: 1st floor plan of Lei family's ancestral hall

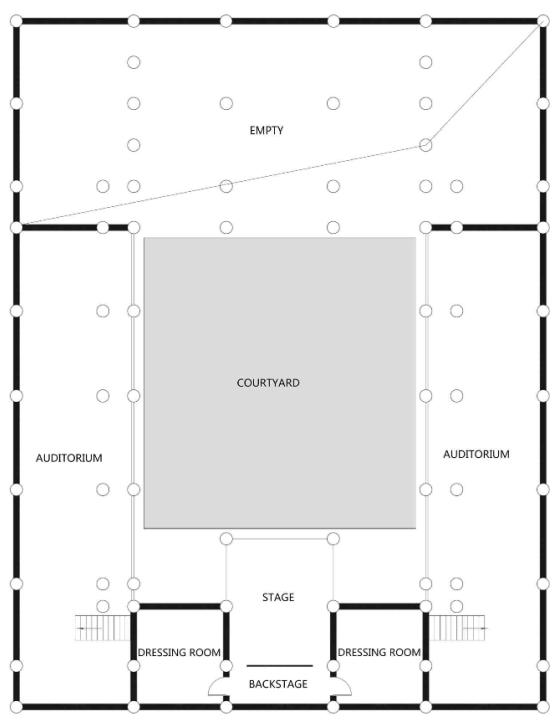

Fig.3: 2nd floor plan of Lei family's ancestral hall 


\section{Architectual space and form of Lei family's ancestral hall stage}

The structure of Lei family's ancestral hall stage is beam frame in wood, and its vertical space can be divided into three parts of stylobate, stage, and roof (Fig.4). The height of stylobate is about 1.6 meters and below the stage is insightful space which constitutes the entryway. The stage has view areas on three sides which can extend the scope of vision and enhance utilization ratio of auditorium. However, the corner posts also block audiences' sight [4]. The stage is supported by wooden pillars. Due to rainy and moist climate in Anhua district, wooden pillars can not directly touch the ground but be fastened on the stone plinth. The height of the stage is about 3.4 meters. To prevent performers on stage from feeling suppressed and to enhance acoustic effects at the same time, at the center of stage ceiling is equipped with caisson to augment the space and enhance performance effect. The stage roof is featured with hip-and-gable roof covered with indigo imbrexes, the overhanging eaves upwarp, which enlarges viewing area and breaks the sense of seriousness in ancestral hall, making the buildings more dynamic and beautiful.

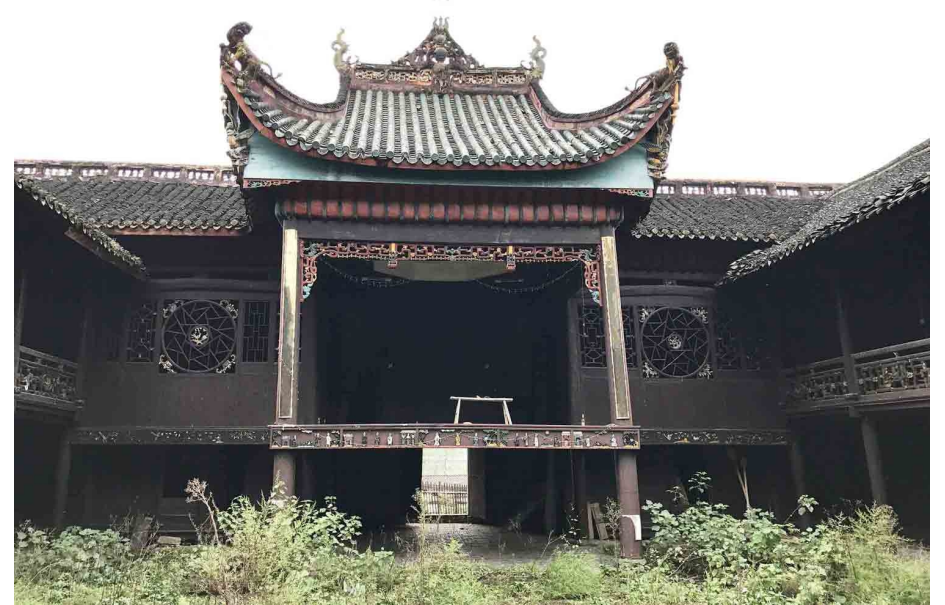

Fig.4 Vertical space of Lei family’s ancestral hall stage

\section{Decorative art of Lei family's ancestral hall stage}

Ancient stage, a special architectural type, is endowed with unique functions and characteristics. It serves as a material carrier of traditional Chinese opera, and various forms of traditional Chinese opera also promote ancient stages to develop its own features in decorating details. Especially when the function of stage turns from "offering sacrifice to ancestors" to "entertaining people", to cater for people's aesthetic demand, magnificent decoration has naturally become an aesthetic orientation for the public. Particularly, when it comes to the period of Ming and Qing Dynasties, decorative art of ancient stage witnessed unprecedented development. Decoration of Lei family's ancestral hall stage presents the typical characteristics of decorative art of ancient stage in that period. Since Lei family's ancestral hall stage is made with wood, wood carving has naturally become the major decorative technique of the stage. In the area facing the audience, the wood-carving decoration is particularly delicate with characters in the traditional Chinese opera. Several plots of the legend of Yue Fei in Hunan opera are illustrated on different parts of the stage. Decorative themes in the whole building are abundant with delicate and vivid patterns. This article mainly explores about the decorative art of beam column, sparrow brace, caisson, balustrades and roof in the stage.

Beam column and sparrow brace. Lei family's ancestral hall stage is supported by pillars at the four corners, which constitute the main performance space of the stage. Since the main function of pillars is load-bearing, there is little decoration on them. Stone plinth under the pillars is the most important part for decoration. which is comprised of upper drum-shaped stone blocks and lower base. Pillars start from the surface of stone plinths that are carved with bas-relief in different patterns, mainly, flowers and plants, birds and beasts. Girder is put on pillar, and sparrow braces are put in place between 
girders and pillars to reduce downward shear force in the joint. As for Lei family's ancestral hall stage, sparrow brace has evolved into "flower cover" (Fig. 5). It is between the upper side of two pillars, and it is carved with hollow crooked patterns with decoration of honeysuckle. On both ends of "flower cover", there are embosses of dragons' heads with bright colors and delicate sculpture. There is colored drawing of two dragons playing with a pearl on the main girder, and the "pearl" in the middle is the Eight Diagrams (Fig. 6). On both ends of secondary girder, bas-relief of dragon, phoenix, lion and goat is carved there, which presents different colors and vivid form. There are girders under the stage and dressing rooms, and on exterior of the girders are inset sidings. It is unusual that many characters in Hunan opera are carved in the surface of sidings (Fig. 7), such as Yue Fei, Liu Bei, Guan $\mathrm{Yu}$, Cao Cao, Bao Gong and so on. All characters are featured with rich facial expressions and bright-color clothes, each one being vivid from all respects. On lateral sidings, natural scenes can be seen that a lion is running quickly and hunting for prey, and birds are dancing among flowers with fluttering wings.
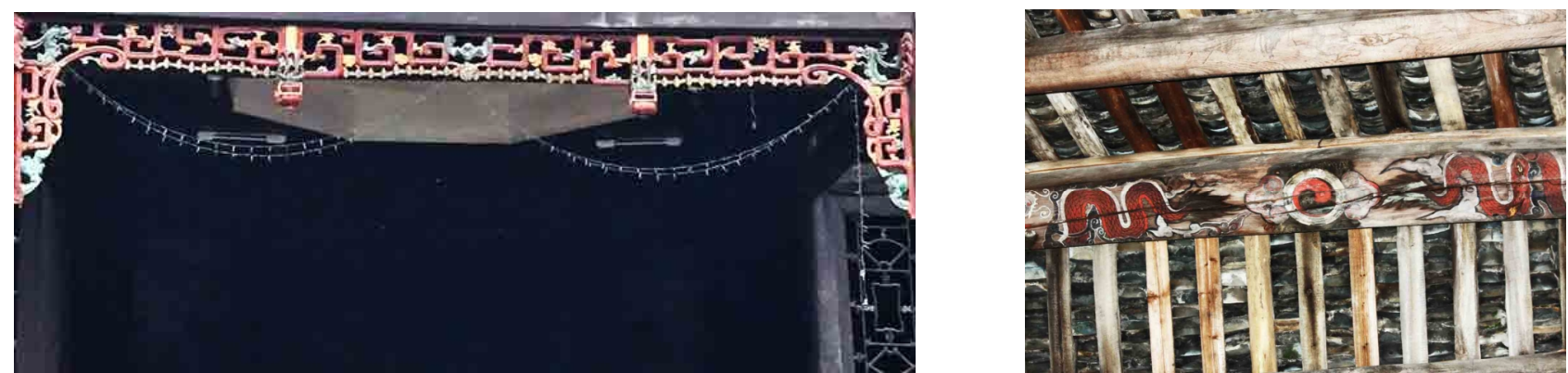

Fig. 5: "Flower cover" of Lei family's ancestral hall stage Fig.
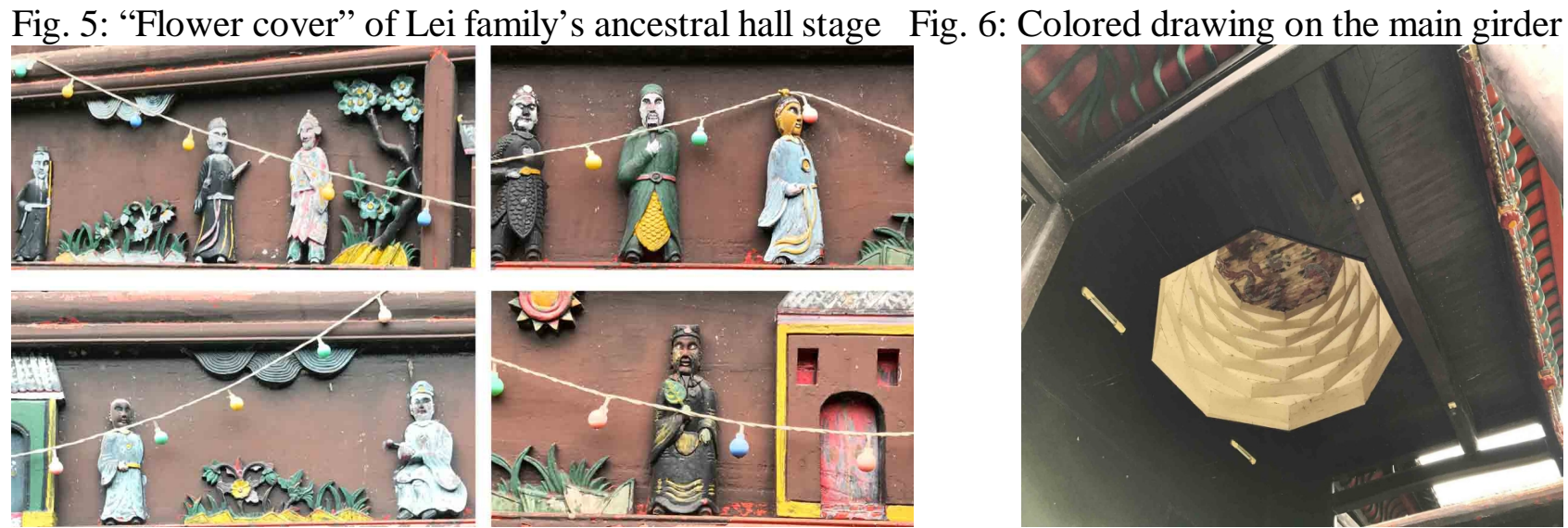

Fig. 7: Characters in Hunan opera in the surface of sidings

Fig. 8: Caisson

Caisson. The caisson, located at ceiling center of the stage (Fig. 8), is a round corbelling caisson, constituted by sixfold of eight corners. It converges at the center, from outside to inside with a form of revolving, with the last layer concentrating on the "bright mirror". Colored drawings on the "bright mirror" (Fig. 9) are four curled-up dragons (which stand for water dragons in the feudal times, therefore they are embedded with implication of fireproof) flying around the eight diagrams in the center. The caisson at the highest place of the stage is designed to enhance the performance effect and space sense of the stage, with curled-up dragon decoration for the wish to prevent fire causing trouble.

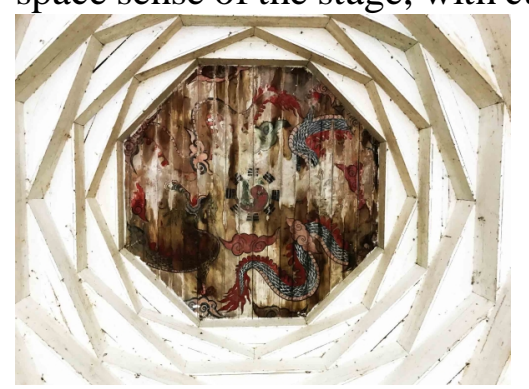

Fig. 9: Colored drawings on the "bright mirror"

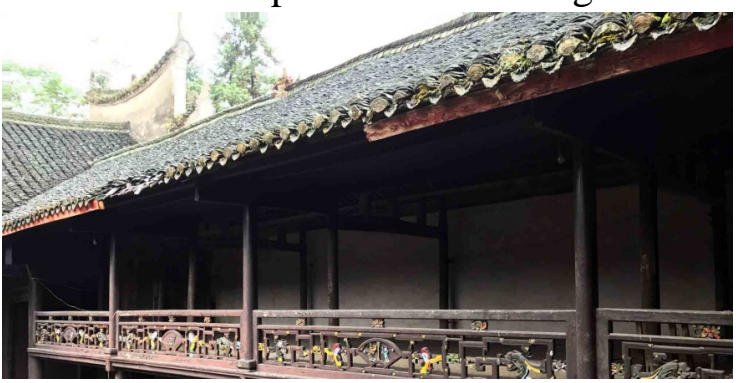

Fig. 10: Balustrades between the pillars 
Balustrades. The auditorium is on the second floor of the ancestral hall which is supported by 6 pillars, being about 16.5 meters long. Balustrades are set between the pillars (Fig. 10), being about 1.2 meters high. The sculptures in balustrades look elegant, and "cloud arches" used for supporting balustrades are decorated with lotus, honeysuckle, phoenix and other patterns with lucky meanings. The balustrades are carved with hollow curving patterns, which are not exactly the same on the two pillars. The centers of balustrades are equipped with fan-shaped planks, and the planks at east balustrades of the stage are carved with the images of rabbits, peacocks, phoenix and other animals. The planks at west balustrades of the stage, however, are carved with story plots of West Journey of Xuanzang, Wu Song Fighting the Tiger, and Jiang Taigong Fishing (Fig. 11). The details of these carvings are dealt with delicately. Besides, the clothes and the movement of characters, feathers of birds and beasts are depicted vividly.
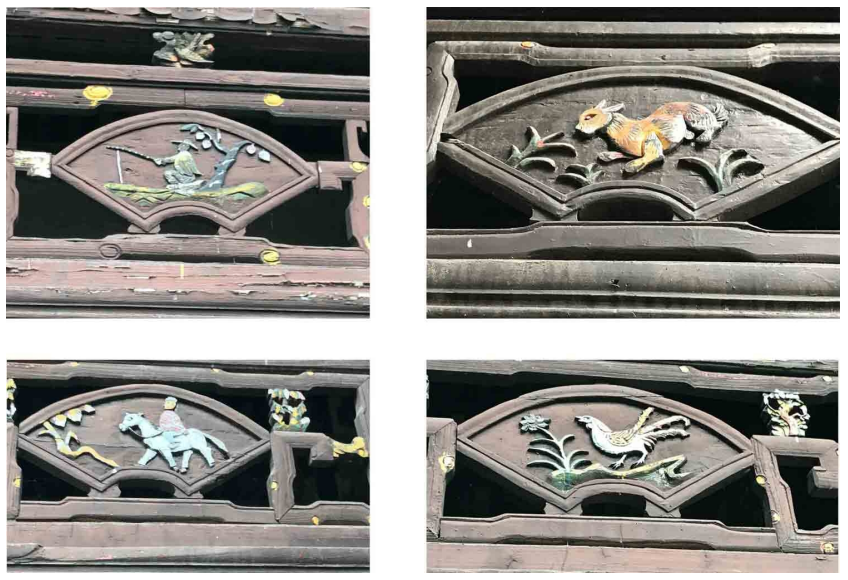

Fig. 11: The patterns in fan-shaped planks at east and west balustrades

Roof. Since the roof has special symbolic significance and good visual effect, the decoration on the stage ridge is also of great significance (Fig.12). The main ridge is a barrel-shaped ridge, and decoration on the middle of the ridge is Aquarius, with three halberds inserted on the top, which commonly means advancing three ranks straightly. The two sides of Aquarius are entwined by two dragons with an open mouth. The Aquarius is a symbol of good fortune and completeness in Buddhism and dragon also represents the meaning of protecting the house. Below the Aquarius, there is a person holding the Aquarius with his hands, with two lions sitting at his two sides. The "swallowing ridge beasts" at the both ends of the ridge are called Chi Wen, which look like fish and dragon. They open up the mouth, and it seems that they would swallow the whole hall ridge, which conveys the meaning of blessing and fire protection. There are four vertical ridges, at the end of which are four diagonal ridges intersected with vertical ridges. The diagonal ridge roofs upwarp, and at the end of diagonal ridges are decorated with a standing lion in the upturned roof-ridges (Fig.13). It seems that the lion would hold the diagonal ridge, with mouth opening and eyes overlooking the whole stage, symbolizing the majesty and braveness. Below the upturned roof-ridge, there is a phoenix as the skew arch, standing on the head of dragon as well as waving the wings that symbolizing the willing and power.

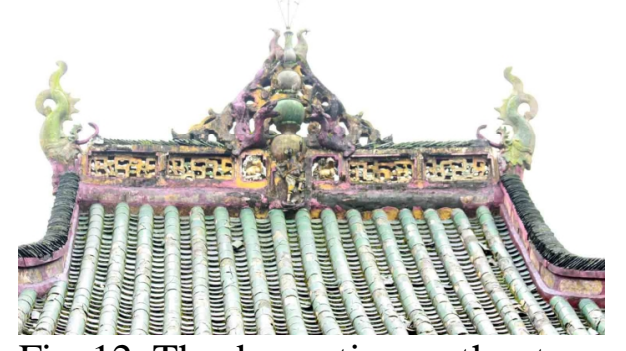

Fig. 12: The decoration on the stage ridge

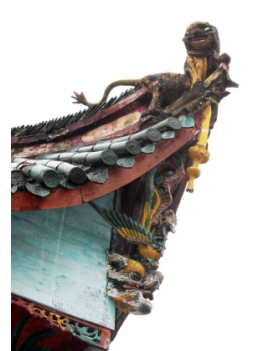

Fig. 13: Standing lion in the upturned roof-ridges 


\section{Value and significance of Lei family's ancestral hall stage}

Lei family's ancestral hall stage is the typical ancient stage in ancestral hall in Meishan area. As a folk public building, it serves the functions of sacrifice, gathering and entertainment. It reflects the wisdom of Meishan people as well as the cohesion and testimony of politics, economy and culture. It is also a material and spiritual treasure. It carries the historical information in specific historical conditions, which has a certain historical and cultural value. The architectural form and the decorative art of the stage reflect the exquisite craftsmanship of the builders at that time, which is worth analyzing and reflecting. It also has a certain scientific and aesthetic value. With the development of contemporary society, many people have neglected the traditional Chinese opera culture, and the stage as the material reflection of traditional Chinese opera is fades away from people's life gradually. Nowadays, most stages in Meishan area are inordinately destroyed, and Lei family's ancestral hall stage in Changtang Town, Anhua County is preserved well and can be used as a case to promote the repair work as well as protection of other ancestral hall stages in Meishan area.

\section{Conclusions}

Currently, the ancestral hall stage in Meishan area is facing a survival challenge. On the one hand, suffering from natural and man-made destruction, ancient stages are seriously damaged or completely disappear. On the other hand, the building is generally abandoned because of the loss of its original functions. The investigation reveals, that although in recent years, people gradually realize the significance of the ancient stage, however, the lack of fund and restoration technology has led to difficulty in carrying out protection. Faced with those problems, I believe that it is imperative to strengthen the intensity and depth of the study over ancient stage and to come up with a more perfect and valuable protection scheme.

\section{References}

[1] Information on http://baike.sogou.com/v7716952.htm

[2] [Yuan Dynasty] Tuo Tuo, 494 collected biographies, History of Song Dynasty Volume-Meishan, Shanghai: Zhonghua Book Publishers 1977, p14194 (In Chinese)

[3] Traditional Chinese opera, Hunan Volume, Beijing: Culture and Publishers (1990), p. 7 (In Chinese)

[4] Deyin Luo, Ancient Stage in China, Nanjing: Southeast University Publishers (2009), p. 65 (In Chinese) 